\title{
CONTINUED FRACTIONS WITH ABSOLUTELY CONVERGENT EVEN AND ODD PARTS
}

\author{
BY \\ R. E. LANE AND H. S. WALL
}

1. Introduction. Several years ago, Scott and Wall [2](1) investigated the continued fraction

$$
f=K_{p=1}^{\infty} \frac{c_{p-1}}{1} \quad\left(c_{0}=1\right)
$$

by means of the system of inequalities

$$
\begin{aligned}
& r_{1}\left|1+c_{1}\right| \geqq\left|c_{1}\right|, \quad r_{2}\left|1+c_{1}+c_{2}\right| \geqq\left|c_{2}\right|, \\
& \quad r_{p}\left|1+c_{p-1}+c_{p}\right| \geqq r_{p} r_{p-2}\left|c_{p-1}\right|+\left|c_{p}\right|, p=3,4,5, \cdots .
\end{aligned}
$$

These are actually the Pringsheim inequalities applied to the even and odd parts of $f$ [2, p. 161]. The $r_{p}$ are nonnegative numbers (depending, in general, upon the $c_{p}$ ), and inequality is required in the first two in case the $c_{p}$ are different from zero. Leighton [1] had employed these inequalities in the case $r_{p}=\left|c_{p}\right| \neq 0$, and had found that $f$ converges if, in addition, $\lim \sup \left|c_{p}\right|$, for $p=\infty$, is finite. Scott and Wall showed that if the $r_{p}$ are subjected to certain restrictions, for example, $r_{p}=1$, or $\lim \inf r_{1} r_{2} \cdots r_{p}=0$, then the divergence of the series $\sum\left|b_{p}\right|$, where $b_{1}=1, c_{p}=1 / b_{p} b_{p+1}, p=1,2,3, \cdots$ (the series to be counted as divergent if some $c_{p}$ vanishes), is necessary and sufficient for the convergence of $f$. In this paper we show that the same conclusion holds without any restruction upon the $r_{p}(\$ 4)$. This is accomplished by showing, more generally, that when the even and odd parts of a continued fraction $f$ are absolutely convergent $(\$ 2)$, then $f$ converges if, and only if, the series $\sum\left|b_{p}\right|$ is divergent. We base our investigations upon the linear fractional transformation $w=T_{p}(z)$ which carries the points $\infty, 0$, and 1 into $f_{p-1}, f_{p}$, and $f_{p+1}$, respectively, where $f_{0}=0, f_{1}=1, f_{2}=1 /\left(1+c_{1}\right), \cdots$ is the sequence of approximants of $f$. Other results in this paper include: a theorem connecting the fixed points of $T_{p}$ with the convergence and value of $f(\$ 2)$, sufficient conditions for absolute convergence of $f(\S \S 3,6)$, and some new convergence regions for $f(\$ 5)$. The latter are found by means of certain geometrical properties of the inequalities (1.1).

2. The transformation $T_{p}$. It will be convenient to introduce the following notations:

$D$ is the set of "points" $c=\left(c_{1}, c_{2}, c_{3}, \cdots\right)$ such that the denominators $B_{0}=1, B_{1}=1, B_{p+1}=B_{p}+c_{p} B_{p-1}, p=1,2,3, \cdots$, of $f=f(c)$ are all different from zero;

Presented to the Society, December 30, 1948; received by the editors December 13, 1948.

(1) Numbers in brackets refer to the bibliography at the end of the paper. 
$D^{*}$ is the subset of points $c=\left(c_{1}, c_{2}, c_{3}, \cdots\right)$ of $D$ such that $c_{p} \neq 0$, $p=1,2,3, \cdots$.

Let $c$ be in $D$. The continued fraction $f(c)$ is said to be absolutely convergent if the series $f_{0}+\sum\left(f_{p}-f_{p-1}\right)$ is absolutely convergent, where $f_{0}, f_{1}, f_{2}, \cdots$ is the sequence of approximants of $f$. The even (odd) part of $f$ is said to be absolutely convergent if the series $f_{0}+\sum\left(f_{2 p}-f_{2 p-2}\right)\left(f_{1}+\sum\left(f_{2 p+1}-f_{2 p-1}\right)\right)$ is absolutely convergent.

It is clear that $f$ and its even and odd parts are absolutely convergent if $c$ is in $D-D^{*}$ (cf., for instance, [4, p. 26]). We therefore assume from now on that $c$ is in $D^{*}$.

Let

$$
t_{p}(z)=\frac{1}{1+c_{p} z}, \quad T_{p}(z)=t_{1} t_{2} \cdots t_{p}(z), \quad p=1,2,3, \cdots .
$$

Since $c$ is in $D^{*}$, the approximants

$$
f_{p-1}=T_{p}(\infty), \quad f_{p}=T_{p}(0), \quad f_{p+1}=T_{p}(1)
$$

are finite. Therefore, if $T_{p}\left(h_{p}\right)=\infty$, then $h_{p}$ is finite and different from 0 and 1 . We observe that

$$
T_{p+1}\left(h_{p+1}\right)=T_{p} t_{p+1}\left(h_{p+1}\right)=T_{p}\left(h_{p}\right)=\infty,
$$

and consequently

$$
h_{p}=t_{p+1}\left(h_{p+1}\right)=\frac{1}{1+c_{p+1} h_{p+1}}, \quad p=1,2,3, \cdots .
$$

Note that $h_{1}=-1 / c_{1}$.

THEOREM 2.1. Let

$$
\begin{array}{r}
w_{1}=-\frac{1}{c_{1}}, \quad w_{p}=\frac{h_{p}}{\left(h_{1}-1\right)\left(h_{2}-1\right) \cdots\left(h_{p-1}-1\right)}, \\
p=2,3,4, \cdots .
\end{array}
$$

Then

$$
T_{p}(z)=f_{p-1}+\frac{w_{p}}{h_{p}-z}, \quad \quad p=1,2,3, \cdots
$$

Proof. Since

$$
T_{1}(z)=t_{1}(z)=0+\frac{-1 / c_{1}}{\left(-1 / c_{1}\right)-z},
$$

the formula is correct for $p=1$. Since $T_{p}(\infty)=f_{p-1}, T_{p}\left(h_{p}\right)=\infty$, it is clear that the formula is correct for a suitable constant $w_{p}$. Now, 


$$
w_{p}=h_{p}\left(f_{p}-f_{p-1}\right)=h_{p}\left[T_{p-1}(1)-T_{p-1}(0)\right]=\frac{h_{p} w_{p-1}}{h_{p-1}\left(h_{p-1}-1\right)}
$$

or

$$
\frac{w_{p}}{h_{p}}=\frac{w_{p-1}}{h_{p-1}} \cdot \frac{1}{h_{p-1}-1}=\frac{1}{\left(h_{1}-1\right)\left(h_{2}-1\right) \cdots\left(h_{p-1}-1\right)},
$$

inasmuch as $w_{1} / h_{1}=1$. This gives the value of $w_{p}$ stated in the theorem.

On putting $z=0$ in formula (2.3) we obtain the following corollary.

Corollary 2.1a. For $n=2,3,4, \cdots$,

$$
\begin{aligned}
f_{n} & =f_{n-1}+\frac{1}{\left(h_{1}-1\right)\left(h_{2}-1\right) \cdots\left(h_{n-1}-1\right)} \\
& =1+\sum_{p=1}^{n-1} \frac{1}{\left(h_{1}-1\right)\left(h_{2}-1\right) \cdots\left(h_{p}-1\right)} .
\end{aligned}
$$

Thus, the continued fraction $f$ is equivalent to the infinite series

$$
1+\sum_{p=1}^{\infty} \frac{1}{\left(h_{1}-1\right)\left(h_{2}-1\right) \cdots\left(h_{p}-1\right)}
$$

in the sense that the nth approximant of $f$ is equal to the sum of the first $n$ terms of the series.

In a similar way we obtain the following corollary.

COROLlaRY 2.1b. The even and odd parts of $f$ are equivalent, in the sense of the preceding corollary, to the infinite series

$$
\sum_{p=1}^{\infty} \frac{h_{2 p-1}}{\left(h_{1}-1\right)\left(h_{2}-1\right) \cdots\left(h_{2 p-1}-1\right)}
$$

and

$$
1+\sum_{p=1}^{\infty} \frac{h_{2 p}}{\left(h_{1}-1\right)\left(h_{2}-1\right) \cdots\left(h_{2 p}-1\right)}
$$

respectively.

From these two corollaries we have, immediately, the following corollary.

COROLLARY 2.1c. If the series $\sum\left|h_{p}\right|$ converges, then the continued fraction $f$ diverges by oscillation, its even and odd parts having finite limits $L_{0}$ and $L_{1}$ such that $\left|L_{0}-L_{1}\right|=\coprod_{p=1}^{\infty}\left|h_{p}-1\right|^{-1}$.

If the two series (2.6) and (2.7) are absolutely convergent and the series $\sum\left|h_{p}\right|$ diverges, then we obviously have

$$
\liminf _{p \rightarrow \infty}\left|\frac{1}{\left(h_{1}-1\right)\left(h_{2}-1\right) \cdots\left(h_{p}-1\right)}\right|=0 \text {. }
$$


From (2.4) it then follows that the values $L_{0}$ and $L_{1}$ of the even and odd parts of $f$, which are the sums of the series (2.6) and (2.7), respectively, are equal to one another, so that $f$ is convergent. On combining this result with Corollary $2.1 \mathrm{c}$, we then have the following theorem.

THEOREM 2.2. If $c$ is in $D^{*}$, and if the even and odd parts of the continued fraction $f(c)$ are absolutely convergent, then $f(c)$ converges if, and only if, the series $\sum\left|h_{p}\right|$ is divergent.

The same type of reasoning used above will show, more generally, that the following theorem is true.

THEOREM 2.2a. If $c$ is in $D^{*}$, if the even and odd parts of $f(c)$ are convergent, the even (odd) part being absolutely convergent, and if the series $\sum\left|h_{2 p-1}\right|$ $\left(\sum\left|h_{2 p}\right|\right)$ diverges, then $f(c)$ converges.

If $c_{p} \neq 0, p=1,2,3, \cdots$, then $f$ may be thrown into the form

$$
\underset{p=1}{\infty} \frac{1}{b_{p}}
$$

where

$$
b_{1}=1, \quad c_{p}=\frac{1}{b_{p} b_{p+1}}, \quad p=1,2,3, \cdots .
$$

We shall now prove the following theorem.

THEOREM 2.3. If $c$ is in $D^{*}$, and if there is a finite constant $M$ such that $\left|f_{p}\right| \leqq M$ for $p=1,2,3, \cdots$, then the two series $\sum\left|h_{p}\right|$ and $\sum\left|b_{p}\right|$ converge or diverge together.

Proof. From the formula (2.1) we find that

$$
h_{p+1}=\frac{1-h_{p}}{h_{p} c_{p+1}}=\frac{1-h_{p}}{h_{p}} \cdot b_{p+1} b_{p+2}
$$

or

$$
b_{p+2}=\frac{h_{p} h_{p+1}}{\left(1-h_{p}\right) b_{p+1}}, \quad \quad p=1,2,3, \cdots
$$

Therefore,

$$
\begin{gathered}
b_{1}=1, \quad b_{2}=\frac{1}{c_{1}}=-h_{1}, \quad b_{3}=-h_{2} \cdot \frac{1}{\left(1-h_{1}\right)}, \\
b_{2 p+2}=-h_{2 p+1} \cdot \frac{\left(1-h_{1}\right)\left(1-h_{3}\right) \cdots\left(1-h_{2 p-1}\right)}{\left(1-h_{2}\right)\left(1-h_{4}\right) \cdots\left(1-h_{2 p}\right)}, \quad p=1,2,3, \cdots . \\
b_{2 p+3}=-h_{2 p+2} \cdot \frac{\left(1-h_{2}\right)\left(1-h_{4}\right) \cdots\left(1-h_{2 p}\right)}{\left(1-h_{1}\right)\left(1-h_{3}\right) \cdots\left(1-h_{2 p+1}\right)},
\end{gathered}
$$


Consequently, if the series $\sum\left|h_{p}\right|$ converges, then the series $\sum\left|b_{p}\right|$ converges. Also,

$$
h_{1}=-b_{2}, \quad h_{2}=-b_{3}\left(1+b_{2}\right), \quad h_{3}=-b_{4} \cdot \frac{1+b_{3}\left(1+b_{2}\right)}{1+b_{2}},
$$

and, by mathematical induction,

$$
h_{p}=-b_{p+1} \cdot \frac{Q_{p}}{Q_{p-1}}, \quad \quad p=1,2,3, \cdots,
$$

where $Q_{0}=Q_{1}=1, Q_{p+1}=b_{p+1} Q_{p}+Q_{p-1}, p=1,2,3, \cdots$, so that $Q_{p}$ is the $p$ th denominator of the continued fraction (2.8). Now, it is well known (cf., for instance, $\left[4\right.$, p. 28]) that when the series $\sum\left|b_{p}\right|$ converges, then $Q_{2 p}$ and $Q_{2 p+1}$ converge, for $p \rightarrow \infty$, to finite limits $K_{0}$ and $K_{1}$. Inasmuch as, by the hypothesis,

$$
\left|f_{n}-f_{n-1}\right|=\left|\frac{1}{Q_{n} Q_{n-1}}\right| \leqq 2 M, \quad n=1,2,3, \cdots,
$$

it follows that $K_{0} \neq 0, K_{1} \neq 0$. Hence, by (2.10), if $\sum\left|b_{p}\right|$ converges, then $\sum\left|h_{p}\right|$ converges.

On combining this result with Theorem 2.2, we obtain the following theorem.

THEOREM 2.4. If $c$ is in $D^{*}$, and if the even and odd parts of $f(c)$ are absolutely convergent, then $f(c)$ converges if, and only if, the series $\sum\left|b_{p}\right|(c f .(2.8)$ and (2.9)) is divergent.

REMARK 1. If we put

$$
\rho_{0}=0, \quad \rho_{p}=\frac{1}{h_{p}-1}, \quad p=1,2,3, \cdots,
$$

then we find from (2.1) that

$$
1+\rho_{p+1}=t_{p+1}\left(1+\rho_{p}\right)=\frac{1}{1+c_{p+1}\left(1+\rho_{p}\right)}, \quad p=0,1,2, \cdots
$$

Since $f_{n}=1+\rho_{1}+\rho_{1} \rho_{2}+\cdots+\rho_{1} \rho_{2} \cdots \rho_{n-1}$, these formulas may be used to compute, in succession, the approximants of $f$.

REMARK 2. Since $\left(\left|b_{p} b_{p+1}\right|\right)^{1 / 2}=1 /\left(\left|c_{p}\right|\right)^{1 / 2} \leqq\left(\left|b_{p}\right|+\left|b_{p+1}\right|\right) / 2$, it follows that a sufficient condition for the divergence of $\sum\left|b_{p}\right|$ is the divergence of $\sum\left(1 /\left(\left|c_{p}\right|\right)^{1 / 2}\right.$.

We conclude this section with a theorem connecting the fixed points of the transformation $T_{p}$ with the convergence and value of the continued fraction $f$. 
Theorem 2.5. Let $c$ be in $D^{*}$, and let $x_{p}$ and $y_{p}$ be the fixed points of the transformation $w=T_{p}(z)$. Let $f(c)$ converge and have the value $x$. Then, if the notation is properly chosen,

$$
x_{p} \rightarrow x \quad \text { and } \quad y_{p}-h_{p} \rightarrow 0,
$$

as $p \rightarrow \infty$.

Conversely, if (2.12) holds, then $f(c)$ converges to the value $x$.

Proof. Since $f_{p-1}=T_{p}(\infty)$ is finite, the fixed points are finite, and since the number $w_{p}$ in (2.3) is not zero, $f_{p-1}$ is not a fixed point. The transformation $w=T_{p}(z)$ carries $y_{p}, h_{p}$, and $\infty$ into $y_{p}, \infty$, and $f_{p-1}$, respectively, and carries $x_{p}, h_{p}$, and $\infty$ into $x_{p}, \infty$, and $f_{p-1}$, respectively. Consequently, $f_{p-1}, x_{p}, h_{p}$, and $y_{p}$ are the four vertices of a parallelogram. Therefore

$$
f_{p-1}=x_{p}+y_{p}-h_{p}
$$

Hence, we may write

$$
T_{p}(z)=x_{p}+y_{p}-h_{p}+\frac{\left(x_{p}-h_{p}\right)\left(y_{p}-h_{p}\right)}{h_{p}-z} .
$$

Since $T_{p}(0)=f_{p}$, this gives

$$
f_{p}=\frac{x_{p} y_{p}}{h_{p}} .
$$

The last statement in the theorem follows immediately from (2.13). By (2.2), (2.3), (2.13), and (2.14),

$$
\begin{aligned}
\frac{\left(x_{p}-h_{p}\right)\left(y_{p}-h_{p}\right)}{h_{p}} & =\frac{\left(x_{p}-h_{p}\right)\left(f_{p-1}-x_{p}\right)}{h_{p}} \\
& =\frac{1}{\left(h_{1}-1\right)\left(h_{2}-1\right) \cdots\left(h_{p-1}-1\right)}
\end{aligned}
$$

Hence, if $f$ converges to a value $x$, we conclude by Corollary 2.1a that for each positive number $\epsilon$ there exists a number $N$ such that

$$
\left|1-\frac{x_{n}}{h_{n}}\right| \cdot\left|f_{n-1}-x_{n}\right|<\epsilon^{2} \quad \text { for } n>N .
$$

On making use of (2.15) we then find that, for any particular $n>N$, one of the following inequalities must hold:

$$
\left|1-\frac{f_{n}}{y_{n}}\right|<\epsilon, \quad \text { or } \quad\left|f_{n-1}-x_{n}\right|<\epsilon .
$$

Therefore, if the notation is appropriately chosen, $x_{p} \rightarrow x$ and by (2.13), $y_{p}-h_{p} \rightarrow 0$.

This completes the proof of Theorem 2.5. 
3. Absolute convergence in the neighborhood of the origin. Let $\gamma$ be a number which is not real and less than or equal to $-1 / 4$. We shall say that $c=\left(c_{1}, c_{2}, c_{3}, \cdots\right)$ is in $V_{\gamma}$ if there exist positive numbers $k_{1}, k_{2}, k_{3}, \cdots$ such that $\left|c_{p}-\gamma\right| \leqq k_{p}, p=1,2,3, \cdots$, and such that $f\left(c^{\prime}\right)$ converges for every $c^{\prime}=\left(c_{1}^{\prime}, c_{2}^{\prime}, c_{3}^{\prime}, \cdots\right)$ such that $\left|c_{p}^{\prime}-\gamma\right| \leqq k_{p}, p=1,2,3, \cdots$. It is known that $V_{\gamma}$ exists when $\gamma$ is restricted as above (cf., for instance, [4, p. 137]). The restriction on $\gamma$ is necessary, since $f(c)$ diverges if $c=(x, x, x, \cdots)$, $-\infty<x<-1 / 4$. If $\gamma=r e^{i \phi},-\pi<\phi<+\pi, r \geqq \cos ^{2} \phi / 2$, it can be shown that a sufficient condition for $c$ to be in $V_{\gamma}$ is that

$$
\left|c_{p}-\gamma\right| \leqq 2 \cos \frac{\phi}{2}\left[r_{1}\left(1-g_{p-1}\right) g_{p}\right]^{1 / 2},
$$

where $0<r_{1}<r, 0<g_{p-1}<1, p=1,2,3, \cdots$. Only when $\gamma=0$ have we a complete characterization of $V_{\gamma}$.

THEOREM 3.1. The point $c=\left(c_{1}, c_{2}, c_{3}, \cdots\right)$ is in $V_{0}$ if, and only if, numbers $g_{0}, g_{1}, g_{2}, \cdots$, greater than zero and less than unity, exist, such that

$$
\left|c_{p}\right| \leqq\left(1-g_{p-1}\right) g_{p}, \quad \quad p=1,2,3, \cdots .
$$

Moreover, if $c$ is in $V_{0}$, then $f(c)$ is absolutely convergent.

REMARK. The condition stated is equivalent to the condition that numbers $m_{p}$ exist such that $0<m_{p}<1,\left|c_{1}\right| \leqq m_{1},\left|c_{p+1}\right| \leqq\left(1-m_{p}\right) m_{p+1}, p=1,2,3, \cdots$, and the series

$$
1+\sum \frac{m_{1} m_{2} \cdots m_{p}}{\left(1-m_{1}\right)\left(1-m_{2}\right) \cdots\left(1-m_{p}\right)}
$$

is convergent (cf. [4, pp. 82-83]).

Proof. One may easily show by mathematical induction, using (2.11), that $\left|c_{1}\right| \leqq m_{1},\left|c_{p+1}\right| \leqq\left(1-m_{p}\right) m_{p+1}, p=1,2,3, \cdots$, implies that

$$
\left|\rho_{p}\right| \leqq \frac{m_{p}}{1-m_{p}}, \quad p=1,2,3, \cdots,
$$

so that when the series (3.2) converges, then $f$ converges absolutely by Corollary 1.1a. This was proved by E. B. Van Vleck [3].

The necessity of the condition can be obtained as follows. Suppose that $c$ is in $V_{0}$. Then there exist positive constants $k_{p}$ such that $\left|c_{p}\right| \leqq k_{p}$ and $f\left(c^{\prime}\right)$ converges for $\left|c_{p}^{\prime}\right| \leqq k_{p}$. In particular, the continued fraction

$$
\frac{1}{1+\frac{k_{1} z}{1+\frac{k_{2} z}{1+}}}
$$


converges for $|z| \leqq 1$, and the continued fraction obtained from this one by replacing any $k_{p}$ by zero converges for $|z| \leqq 1$. The last statement means that the poles of the approximants are in the domain $|z|>1$. This continued fraction is of the type which figures in the work of Stieltjes. The poles of the approximants are all real, simple, negative, and have positive residues. From the preceding, the poles are all less than -1 . By a well known argument, it follows that the continued fraction is equal to an integral of the form $\int_{0}^{1} d \phi(u) /(1+z u)$, where $\phi(u)$ is bounded and nondecreasing. Application of a theorem of Wall $\left[4\right.$, p. 263] then shows that $k_{p}=\left(1-g_{p-1}\right) g_{p}$, where $0<g_{p-1}$ $<1, p=1,2,3, \cdots$, as was to be proved.

4. Convergence problem for the domain $S$. We shall say that $c=\left(c_{1}, c_{2}, c_{3}, \cdots\right)$ is in the domain $S$ if there exist nonnegative numbers $r_{1}, r_{2}, r_{3}, \cdots$ such that the inequalities (1.1) hold, it being agreed that inequality shall hold in the first two relations if $c_{p} \neq 0, p=1,2,3, \ldots$. (If $c$ is in $S$ and no $c_{p}$ vanishes, we shall say that $c$ is in $S^{*}$.) If $c$ is in $S$, then $c$ is in $D$ [4, p. 41]. Obviously, if $c$ is in $S^{*}$, then the numbers $r_{p}$ are all positive.

LEMMA 4.1. If $c$ is in $S$, then the even and odd parts of $f(c)$ are absolutely convergent.

Proof. The case where $c$ is in $S-S^{*}$ is covered by the statement near the beginning of $\$ 2$. If $c$ is in $S^{*}$, then the even and odd parts of $f(c)$ can be thrown into the form $K\left(c_{p-1}^{\prime} / 1\right) \quad\left(c_{0}^{\prime}=1\right)$, multiplied by an unimportant factor plus an additive term, where $c^{\prime}=\left(c_{1}^{\prime}, c_{2}^{\prime}, c_{3}^{\prime}, \cdots\right)$ is in $V_{0}(\S 3)$ $[4$, p. 54] and are therefore absolutely convergent by Theorem 3.1 .

THEOREM 4.1. If $c$ is in $S$, then $f(c)$ converges if, and only if, (a) $c$ is in $S-S^{*}$, or (b) $c$ is in $S^{*}$, and the series $\sum\left|b_{p}\right|$, defined by (2.9), is divergent.

Proof. Convergence of $f(c)$ in case (a) is covered by the remark near the beginning of $\S 2$. The rest follows immediately from Lemma 4.1 and Theorem 2.4 .

This result includes or extends several of the theorems of [2], particularly Theorem 3.3, p. 161, Theorem 3.4 and Theorem D, p. 163, and Theorem E, p. 165.

5. Geometrical characterization of $S$. Let $c_{1}, c_{2}, c_{3}, \cdots$ be complex numbers different from zero, and let $r_{0}, r_{1}, r_{2}, \cdots$ be positive numbers. The following regions of the $z$-plane have been determined in such a way that

$$
\begin{aligned}
& t_{p}\left(H_{p}\right)=R_{p-1}, \quad t_{p+1}\left(G_{p+1}\right)=H_{p}, \quad t_{p} t_{p+1}\left(G_{p+1}\right)=R_{p-1}, \\
& p=1,2,3, \cdots \text {; } \\
& R_{p}: \quad|z-1| \leqq 1 / r_{p} \\
& p=0,1,2, \cdots \text {; } \\
& H_{p}: \quad\left|z+\frac{1}{c_{p}}\right| \geqq r_{p-1}|z|, \\
& p=1,2,3, \cdots \text {; } \\
& G_{p}: \quad\left|z+\frac{1+c_{p-1}}{c_{p}}\right| \geqq r_{p-2}\left|\frac{c_{p-1}}{c_{p}}\right|, \\
& p=2,3,4, \cdots
\end{aligned}
$$


LEMmA 5.1. For each integer $p \geqq 2$, the inequality

$$
r_{p}\left|1+c_{p-1}+c_{p}\right| \geqq r_{p-2} r_{p}\left|c_{p-1}\right|+\left|c_{p}\right|
$$

is necessary and sufficient in order that

$$
t_{p-1} t_{p}\left(R_{p}\right) \subset R_{p-2}
$$

Proof. Since $t_{p-1} t_{p}\left(G_{p}\right)=R_{p-2}$, a necessary and sufficient condition for (5.3) is $R_{p} \subset G_{p}$. The latter holds if, and only if, the center 1 of $R_{p}$ is in $G_{p}$ at a distance at least $1 / r_{p}$ from the boundary of $G_{p}$ :

$$
\left|1+\frac{1+c_{p-1}}{c_{p}}\right|-\frac{1}{r_{p}} \geqq r_{p-2}\left|\frac{c_{p-1}}{c_{p}}\right|,
$$

that is, if, and only if, (5.2) holds.

LEммA 5.2. For each integer $p \geqq 1$,

$$
t_{p}\left(R_{p}\right) \subset R_{p-1}
$$

if, and only if,

$$
\begin{array}{lll}
\text { (a) } & r_{p}\left|c_{p}+\frac{1}{1-r_{p-1}^{2}}\right| \geqq \frac{r_{p-1} r_{p}}{1-r_{p-1}^{2}}+\left|c_{p}\right| & \text { for } r_{p-1}<1, \\
\text { (b) } & r_{p}\left(1+c_{p}+\bar{c}_{p}\right) \geqq 2\left|c_{p}\right| & \text { for } r_{p-1}=1, \\
\text { (c) } & r_{p}\left|c_{p}+\frac{1}{1-r_{p-1}^{2}}\right| \leqq \frac{r_{p-1} r_{p}}{r_{p-1}^{2}-1}-\left|c_{p}\right| & \text { for } r_{p-1}>1
\end{array}
$$

Proof. Since $t_{p}\left(H_{p}\right)=R_{p-1}$, (5.4) holds if, and only if, $R_{p} \subset H_{p}$, and (5.5) is necessary and sufficient for the latter.

LEMMA 5.3. For each integer $p \geqq 1,(5.5)$ implies that

$$
r_{p}\left|1+c_{p}\right| \geqq\left|c_{p}\right|+\frac{r_{p} r_{p-1}}{1+r_{p-1}}>\left|c_{p}\right| .
$$

Proof. If (5.5)(a) holds, then

$$
\begin{aligned}
r_{p}\left|1+c_{p}\right|+\frac{r_{p} r_{p-1}^{2}}{1-r_{p-1}^{2}} & \geqq r_{p}\left|1+c_{p}+\frac{r_{p-1}^{2}}{1-r_{p-1}^{2}}\right| \\
& =r_{p}\left|c_{p}+\frac{1}{1-r_{p-1}^{2}}\right| \geqq\left|c_{p}\right|+\frac{r_{p} r_{p-1}}{1-r_{p-1}^{2}},
\end{aligned}
$$

or (5.6). If (5.5)(b) holds, then 


$$
\begin{aligned}
r_{p}\left|1+c_{p}\right| & \geqq r_{p}\left(1+\frac{c_{p}+\bar{c}_{p}}{2}\right)=\frac{r_{p}}{2} p\left(1+c_{p}+\bar{c}_{p}\right)+\frac{r_{p} r_{p-1}}{1+r_{p-1}} \\
& \geqq\left|c_{p}\right|+\frac{r_{p} r_{p-1}}{1+r_{p-1}} .
\end{aligned}
$$

If (5.5) (c) holds, then

$$
\begin{aligned}
r_{p}\left|1+c_{p}\right| & =r_{p}\left|\frac{r_{p-1}^{2}}{r_{p-1}^{2}-1}+c_{p}-\frac{1}{r_{p-1}^{2}-1}\right| \\
& \geqq \frac{r_{p} r_{p-1}^{2}}{r_{p-1}^{2}-1}-r_{p}\left|c_{p}+\frac{1}{1-r_{p-1}^{2}}\right| \\
& \geqq \frac{r_{p} r_{p-1}^{2}}{r_{p-1}^{2}-1}+\left|c_{p}\right|-\frac{r_{p} r_{p-1}}{r_{p-1}^{2}-1} \\
& =\left|c_{p}\right|+\frac{r_{p} r_{p-1}}{r_{p-1}+1}>\left|c_{p}\right| .
\end{aligned}
$$

Theorem 5.1. Let $c_{p} \neq 0, p=1,2,3, \cdots$. The point $c=\left(c_{1}, c_{2}, c_{3}, \cdots\right)$ is in $S^{*}(c f . \S 4)$ if, and only if, positive numbers $r_{0}, r_{1}, r_{2}, \cdots$ exist such that

$$
t_{1}\left(R_{1}\right) \subset R_{0}, \quad t_{p-1} t_{p}\left(R_{p}\right) \subset R_{p-2}, \quad \quad p=2,3,4, \cdots .
$$

Proof. If (5.7) holds, then (5.2) and (5.6) hold, so that $c$ is in $S^{*}$. Conversely, if $c$ is in $S^{*}$, we may obviously determine $r_{0}$ such that $0<r_{0}<1$, and such that (5.2) holds for $p \geqq 2$ and (5.5) (a) holds with $p=1$, so that, by Lemmas 5.2 and 5.1, the relation (5.7) is satisfied.

Corollary 5.1a. A sufficient condition for $c=\left(c_{1}, c_{2}, c_{3}, \cdots\right)$, where $c_{p} \neq 0, p=1,2,3, \cdots$, to be in $S^{*}$ is that there exist positive numbers $r_{0}, r_{1}, r_{2}, \cdots$ such that

$$
t_{p}\left(R_{p}\right) \subset R_{p-1}, \quad \quad p=1,2,3, \cdots .
$$

Proof. If (5.8) holds, then $t_{1}\left(R_{1}\right) \subset R_{0}, t_{p-1} t_{p}\left(R_{p}\right) \subset t_{p-1}\left(R_{p-1}\right) \subset R_{p-2}$, $p=2,3,4, \cdots$, so that (5.7) holds, and therefore $c$ is in $S^{*}$.

CoRollary 5.1b. If $c$ is in $S^{*}$, then all the approximants of $f(c)$ have their values in $R_{0}$.

Proof. By (5.7), $T_{p}\left(R_{p}\right)=t_{1} t_{2} \cdots t_{p}\left(R_{p}\right) \subset R_{0}$, so that, since 1 is in $R_{p}$, $f_{p+1}=T_{p}(1)$ is in $R_{0}(p=1,2,3, \cdots)$ and $f_{1}=1$.

THEOREM 5.2. A sufficient condition for $c=\left(c_{1}, c_{2}, c_{3}, \cdots\right)$ to be in $S$ is that positive numbers $r_{0}, r_{1}, r_{2}, \cdots$ exist such that (5.5) hold for $p=1,2,3, \cdots$. 
Proof. If $c_{p} \neq 0, p=1,2,3, \cdots$, then, by Lemma 5.2 and Corollary 5.1a, $c$ is in $S^{*} C S$. If some $c_{p}$ vanishes, put $c_{p}^{\prime}=c_{p}+d_{p}$, where $d_{p}=0$ if $c_{p} \neq 0$, and $d_{p} \neq 0,\left|d_{p}\right| \leqq \delta_{p}$, a small positive number, if $c_{p}=0$. Then, (5.5) will hold with the $c_{p}$ replaced by the $c_{p}^{\prime}$ provided the $\delta_{p}$ are sufficiently small. Hence, by the preceding, $c^{\prime}=\left(c_{1}^{\prime}, c_{2}^{\prime}, c_{3}^{\prime}, \cdots\right)$ is in $S$, so that the inequalities (1.1) hold with the $c_{p}$ replaced by the $c_{p}^{\prime}$. Since this is true for all sufficiently small $\delta_{p}$, it is true when the $d_{p}$ are all set equal to zero, that is, $c$ is in $S$.

By Theorem 4.1 we now have the following corollary.

Corollary 5.2a. If (5.5) holds with $r_{p}>0, p=0,1,2, \cdots$, then $f(c)$ converges if, and only if, (a) some $c_{p}$ vanishes or (b) $c_{p} \neq 0, p=1,2,3, \cdots$, and the series $\sum\left|b_{p}\right|$ defined by (2.9) is divergent.

EXAMPLE 1. Let $r_{2 p}=1, p=0,1,2, \cdots$. Then (5.5) becomes

$$
\begin{array}{ll}
r_{2 p-1}\left(1+c_{2 p-1}+\bar{c}_{2 p-1}\right) \geqq 2\left|c_{2 p-1}\right|, & \\
\left(1+c_{2 p}+\bar{c}_{2 p}\right) \geqq 2\left|c_{2 p}\right| & \text { for } r_{2 p-1}=1, \\
\left|c_{2 p}+\frac{1}{1-r_{2 p-1}^{2}}\right| \geqq \frac{r_{2 p-1}}{1-r_{2 p-1}^{2}}+\left|c_{2 p}\right| & \text { for } r_{2 p-1}<1, \\
\left|c_{2 p}+\frac{1}{1-r_{2 p-1}^{2}}\right| \leqq \frac{r_{2 p-1}}{r_{2 p-1}^{2}-1}-\left|c_{2 p}\right| & \text { for } r_{2 p-1}>1, \\
& p=1,2,3, \cdots .
\end{array}
$$

Thus, $c_{2 p-1}$ is in an ellipse and $c_{2 p}$ in a region bounded by a branch of a hyperbola if $r_{2 p-1}<1 ; c_{2 p-1}$ and $c_{2 p}$ are in the parabolic region $|z|-\Re(z) \leqq 1 / 2$ if $r_{2 p-1}=1 ; c_{2 p-1}$ is in a region bounded by a branch of a hyperbola and $c_{2 p}$ is in an ellipse if $r_{2 p-1}>1$. The condition for convergence of $f(c)$ is given by Corollary 5.2a. If $r_{2 p-1}=1$, for $p=1,2,3, \cdots$, this gives the parabola theorem [2]. For any choice of $r_{2 p-1}>0, p=1,2,3, \cdots$, the approximants of (c) have their values in $R_{0}:|z-1| \leqq 1$.

EXAMPLE 2. Let $r_{2 p}=1, p=0,1,2, \cdots$, and let $r_{2 p-1}=\left|c_{2 p-1}\right| / s_{p}, s_{p}>0$, $p=1,2,3, \cdots$. Then $(5.5)$ becomes

$$
\begin{array}{cc}
\Re\left(c_{2 p-1}\right) \geqq\left(2 s_{p}-1\right) / 2, & \text { for }\left|c_{2 p-1}\right|=s_{p}, \\
1+c_{2 p}+\bar{c}_{2 p} \geqq 2\left|c_{2 p}\right| & \text { for }\left|c_{2 p-1}\right|<s_{p}, \\
\left|c_{2 p}+\frac{s_{p}^{2}}{s_{p}^{2}-\left|c_{2 p-1}\right|^{2}}\right| \geqq \frac{s_{p}\left|c_{2 p-1}\right|}{s_{p}^{2}-\left|c_{2 p-1}\right|^{2}}+\left|c_{2 p}\right| & \text { for }\left|c_{2 p-1}\right|>s_{p}, \\
\left|c_{2 p}+\frac{s_{p}^{2}}{s_{p}^{2}-\left|c_{2 p-1}\right|^{2}}\right| \leqq \frac{s_{p}\left|c_{2 p-1}\right|}{\left|c_{2 p-1}\right|^{2}-s_{p}^{2}}-\left|c_{2 p}\right| & p=1,2,3, \cdots .
\end{array}
$$


Thus, for each $p, c_{2 p-1}$ is in a certain half-plane, and $c_{2 p}$ is in a domain bounded by a parabola, a branch of a hyperbola, or an ellipse, according as $\left|c_{2 p-1}\right|=s_{p}$, $<s_{p}$, or $>s_{p}$, respectively. Let $A_{s}$ denote the set of all points $c=\left(c_{1}, c_{2}, c_{3}, \cdots\right)$ satisfying these inequalities, for a given sequence of positive numbers $s_{0}, s_{1}, s_{2}, \cdots$, and $A_{s}^{*}$ the subset of $A_{s}$ such that $c_{p} \neq 0, p=1,2,3, \cdots$. Of course, $A_{s}^{*} \subset S^{*} \subset S$. If $c$ is in $A_{s}-A_{s}^{*}$, then, for each positive number $\epsilon$, we may determine $c^{\prime}$ in $A_{s}^{*}$ such that $\left|c_{p}-c_{p}^{\prime}\right|<\epsilon, p=1,2,3, \cdots$. It follows immediately that $c$ is in $S$. The condition for convergence of $f(c)$ for $c$ in $A_{s}$ is therefore given by Corollary 5.2a. The values of the approximants are all in the domain $R_{0}:|z-1| \leqq 1$.

EXAmple 3. Let $r_{p}=\left|c_{p}\right| / s_{p}, s_{p}>0, p=0,1,2, \cdots,\left(c_{0}=1\right)$. In this case, (5.5) yields for the domain of $c_{p}$ a finite closed circular region, the exterior and boundary of a finite circle or a half-plane plus its boundary, depending upon the value of $c_{p-1}$. By an argument analogous to that used in Example 2, the value $c_{p}=0$ is allowable. The condition for convergence is given by Corollary 5.2a. The values of the approximants are in $R_{0}:|z-1| \leqq s_{0}$.

6. Absolute convergence in the domain $S$. The continued fraction $f(c)$ may be convergent but not absolutely convergent for $c$ in $S$. For example, $c=\left(1^{2}, 2^{2}, 3^{2}, \cdots\right)$ is in $S$ and $f(c)$ is equivalent, in the sense of Corollary 2.1a, to the nonabsolutely convergent series $\sum(-1)^{p-1} / p$. We shall obtain a sufficient condition for $f(c)$ to be absolutely convergent for $c$ in $S$. We first prove the following lemma.

Lemma 6.1. If $c=\left(c_{1}, c_{2}, c_{3}, \cdots\right)$ is in $S^{*}$, then (cf. $\left.\S 2\right)$

$$
\left|\frac{1}{h_{p}}\right|<\left|c_{p}\right|\left(1+r_{p-1}\right), \quad p=1,2,3, \cdots .
$$

Proof. Since $1 / h_{1}=-c_{1}$ (cf. $\S 2$ ), the inequality is true for $p=1$. By (5.7), $T_{p}\left(R_{p}\right)=t_{1} t_{2} \cdots t_{p}\left(R_{p}\right) \subset R_{0}$, so that, since $T_{p}\left(h_{p}\right)=\infty, h_{p}$ is outside $R_{p}$ :

$$
\left|h_{p}-1\right|>1 / \dot{r}_{p}, \quad p=1,2,3, \cdots \text {. }
$$

Let us suppose that, for a particular value of $p>1$, (6.1) fails to hold; that is,

$$
\left|\frac{1}{h_{p}}\right| \geqq\left|c_{p}\right|\left(1+r_{p-1}\right) .
$$

Then, by (2.1) and (6.2), we get

$$
\left|c_{p}\right|=\left|\frac{1-h_{p-1}}{h_{p-1} h_{p}}\right|>\left|\frac{1}{h_{p-1} h_{p}}\right| \cdot \frac{1}{r_{p-1}} \geqq\left|\frac{1}{h_{p-1}}\right| \cdot \frac{\left|c_{p}\right|\left(1+r_{p-1}\right)}{r_{p-1}},
$$

or

$$
\left|\frac{1}{h_{p-1}}\right|<\frac{r_{p-1}}{1+r_{p-1}} .
$$

Therefore 


$$
\left|c_{p}\right|=\left|1-\frac{1}{h_{p-1}}\right| \cdot\left|\frac{1}{h_{p}}\right|>\left(1-\frac{r_{p-1}}{1+r_{p-1}}\right) \cdot\left|c_{p}\right|\left(1+r_{p-1}\right),
$$

which is impossible. This contradiction establishes the lemma.

THEOREM 6.1. Let $r, K$, and $N$ be positive constants. Suppose that $c=\left(c_{1}, c_{2}, c_{3}, \cdots\right)$ is in $S^{*}$, the inequalities (1.1) holding with $r_{p} \leqq r$, $p=1,2,3, \cdots$, and that $\left|c_{p}\right| \leqq K$ for $p=p_{1}, p_{2}, p_{3}, \cdots$, where $0<p_{k+1}$ $-p_{k} \leqq N, k=1,2,3, \cdots$. Then $f(c)$ is absolutely convergent.

Proof. Let $m$ denote any one of the indices $p_{k}$. By (6.1),

$$
\left|h_{m}\right|>\frac{1}{\left|c_{m}\right|\left(1+r_{m-1}\right)} \geqq \frac{1}{K(1+r)} .
$$

By Lemma 4.1, the series (2.6) and (2.7) are absolutely convergent. Therefore, by $(6.3)$, the series

$$
\sum_{(p)} \frac{1}{\left(h_{1}-1\right)\left(h_{2}-1\right) \cdots\left(h_{p}-1\right)} \quad\left(p=p_{1}, p_{2}, p_{3}, \cdots\right)
$$

is absolutely convergent. Since, by (6.2)

$$
\left|\frac{1}{h_{p}-1}\right|<r_{p} \leqq r,
$$

it follows that the $N$ series of this kind obtained by summing over the indices $p=p_{1}+s, p_{2}+s, p_{3}+s, \cdots$, for $s=1,2,3, \cdots, N$, are absolutely convergent. On adding together the series (6.4) and these $N$ series, we obtain an absolutely convergent series containing all the terms of the series (2.5). Therefore the latter series is absolutely convergent, and the theorem is established.

Corollary 6.1a. If $\left|c_{p}\right|-\Re\left(c_{p}\right) \leqq 1 / 2, p=1,2,3, \cdots$, and if $\left|c_{p}\right| \leqq K$ for the values of $p$ specified in Theorem 6.1, then $f(c)$ is absolutely convergent.

In this case (1.1) holds with $r_{p}=1$ and with inequality in the first two relations.

\section{BIBLIOGRAPHY}

1. Walter Leighton, Sufficient conditions for the convergence of a continued fraction, Duke Math. J. vol. 4 (1938) pp. 775-778.

2. W. T. Scott and H. S. Wall, A convergence theorem for continued fractions, Trans. Amer. Math. Soc. vol. 47 (1940) pp. 155-172.

3. E. B. Van Vleck, On the convergence and character of the continued fraction ..., Trans. Amer. Math. Soc. vol. 2 (1901) pp. 476-483.

4. H. S. Wall, Analytic theory of continued fractions, New York, 1948.

UnIversity of TeXas, Austin, Tex. 